\title{
Energetic Approach for Control Surface Disconnection Fault Detection in Hydraulic Aircraft Actuators
}

\author{
Lörinc Márton* Daniel Ossmann* \\ * Robotics and Mechatronics Center, DLR - German Aerospace \\ Center, Münchenerstr. 20, D-82234 Weßling, Germany \\ (e-mails: martonl@ms.sapientia.ro (Marton.Loerinc@dlr.de), \\ Daniel.Ossmann@dlr.de).
}

\begin{abstract}
This work proposes a new approach to detect the disconnected mechanical load in electro-hydraulically actuated control systems. The detector applies a Fault Model of the hydraulic actuator in which the load force is neglected. Instead of system output based analytical redundancy the model compares the energy intake of the real actuator and of the Fault Model. With this approach the disconnection between the load and the actuator can be detected, even when the difference between the real system output and Fault Model output is in order of the measurement noise. The proposed method is applicable for aircraft control surface disconnection detection with unmeasurable aerodynamic force.
\end{abstract}

Keywords: Fault detection, Energy balance, Hydraulic actuators, Physical models, Aircraft control

\section{INTRODUCTION}

The control surfaces of most of transport aircrafts are hydraulically powered. The servo controlled electro-hydraulic actuation represents the classical approach to steer the control surfaces. These actuators are one of the key elements for the implementation of fly-by-wire digital aircraft control systems.

Duplication of control surfaces with same roles on commercial aircrafts provides control system redundancy, making the flight control system reconfigurable. However for the implementation of the reconfigurable control laws the detection of the actuator's dysfunctions is necessary.

During their operation, the electrical and hydraulic actuators, which work in hazardous environments, are exposed to various types of faults (mechanical disconnection of the load, leakage of the hydraulic fluid, overload, etc.). Reliable detection of these faults is indispensable especially in safety critical systems such as aircrafts. The classical approach for detecting faults is to run a reliable process model parallel with the real process with same inputs (analytical redundancy). Based on the difference between the real output and the model output a residual signal is generated. If the residual is greater than a given threshold value, the system is in fault mode.

The mathematical model of the electro-hydraulic actuators are inherently nonlinear, hence nonlinear fault detection techniques (Garcia and Frank, 1997) can be applied in order to achieve reliable detection. Since the model of these actuators can be written in Linear Parameter Varying (LPV) form, the LPV fault detection techniques are suitable for residual generator design for these systems, see e.g. (Varga, 2011). Nonlinear analytical redundancy technique based fault detection and isolation methods for precisely known nonlinear hydraulic actuator model was derived in (Leuschen et al., 2003). Nonlinear observer based fault detection method using simplified first order actuator model was proposed in (Garcia et al., 2009). By applying more complex actuator models, nonlinear observers were also proposed in (Khan et al., 2005). In the work (Tan and Sepehri, 2002) the residual generator design is based on a Volterra model approximation of the hydraulic actuator model. Extended Kalman Filter techniques are also applicable for fault detection in electro-hydraulic actuators (Wang and Syrmos, 2008). On-line parameter estimation approaches for fault detection for hydraulic actuators with uncertain models were presented in the paper (Garimella and Yao, 2005).

In this work a novel fault detection approach is proposed based on the energy balance of the electro-hydraulic actuator. It is assumed that only the rod position of the electro-hydraulic actuator is measurable and the load force that acts on the actuator dynamics is unknown. The proposed approach is applicable even in such cases when the difference between the actual actuator's trim position and the new reference position are close to each other, and the difference between the model output (applied for fault detection) and the real output of the system is comparable with the precision of the position sensor or the measurement noise.

Only a few earlier papers deal with the problem of energy balance based fault detection and isolation. In the paper (Chen et al., 2010) an energy balance based fault detection scheme was proposed for a class of linear dissipative systems. In the work (Fantuzzi and Secchi, 2004) fault detection and isolation method was proposed for port- 
Hamiltonian systems to detect parameter variations. The energy balance based fault detection was also applied for sensor fault detection in steel galvanizing process, see the method proposed in (Theilliol et al., 2006).

The paper is organized as follows: Section 2 details the model of the actuator. It is presented, how the simple first order electro-hydraulic actuator model, applicable for aircraft control design and for fault detection, can be derived from basic physical laws. In Section 3 the design of the residual generator for control surface disconnection type fault is presented. Simulation results are provided in Section 4. Finally, this work is concluded in Section 5.

\section{MATHEMATICAL MODEL OF VALVE-CONTROLLED HYDRAULIC ACTUATORS}

The mathematical model that relates the control input (i) to the actuator's rod position $(x)$ depends on the dynamics of the valve spool, the nonlinear flows through the valve control ports, the compressibility of fluid flows, as well as the mechanics of the piston motion. The actuator requires an external hydraulic power supply to generate the pressure necessary for driving the piston.

The dynamics of the valve spool can be approximated with a stable linear dynamics with unit amplification. The input of the electric servo is the control signal for the actuator $(i)$, the output is the position of the servo $\left(x_{S}\right)$. Both first order and second order dynamics can be used to describe the servo behavior. In this work the first order model is used with the time constant $T_{S}$ as follows:

$$
T_{S} \dot{x}_{S}=-x_{S}+i \text {. }
$$

The pressure difference $\left(\Delta P=P_{1}-P_{2}\right)$ dynamics in the actuator cylinder is given by (see e.g. (Merritt, 1967)):

$$
C_{H}(x) \Delta \dot{P}=Q_{f}-S \dot{x},
$$

where $Q_{f}$ denotes the flow in the hydraulic circuit and $C_{H}(x)$ is the hydraulic capacity that can be calculated as: $\frac{1}{C_{H}(x)}=\frac{1}{C_{1}(x)}+\frac{1}{C_{2}(x)} ; C_{1}(x)=\frac{V_{01}+S x}{B} ; C_{2}(x)=$ $\frac{V_{02}+S(L-x)}{B}$. Parameters: $V_{01}, V_{02}$ initial chamber volumes, $B$ bulk modulus of the hydraulic fluid, $L$ length of the actuator cylinder.

A simplified model for the flow $Q_{f}$ can be determined based on Bernoulli principle:

$$
Q_{f}=C_{v} x_{S} \sqrt{\frac{2}{\rho}\left(\Delta P_{e x t}-\Delta P\right)},
$$

where $\Delta P_{\text {ext }}$ denotes the nominal pressure of the hydraulic power supply. The parameter $C_{v}$ denotes the coefficient of discharge, $\rho$ is the hydraulic fluid density.

The motion of the rod is described by the following equation:

$$
M \ddot{x}+F_{f}+F_{L}=S \Delta P,
$$

where $x$ denote the rod position, $\Delta P$ is the pressure difference between the two chambers of the actuator, $F_{L}$ refers to the external load, $F_{f}$ is the friction induced damping. Parameters: $S$ is the area of the piston surface, $M$ is the mass of the rod and the load combined.

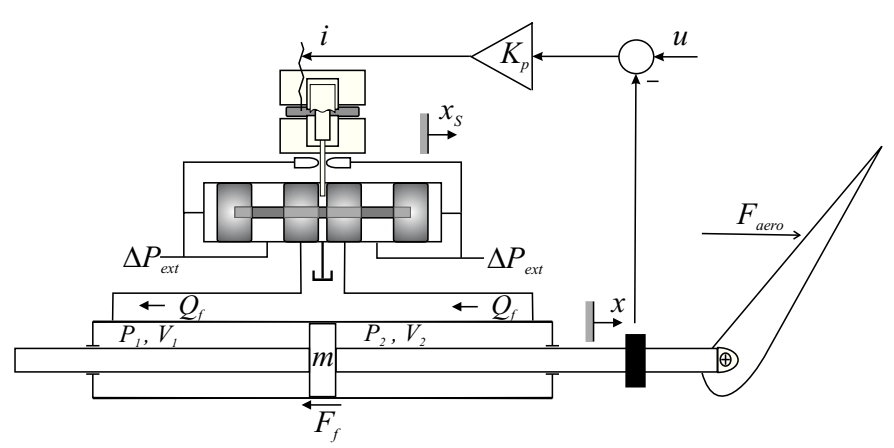

Fig. 1. Hydraulic actuator with aerodynamic force as load

The friction in the model depends on the velocity of the rod $(\dot{x})$. For precise friction modeling in hydraulic actuators nonlinearly parameterized models can be applied (Márton et al., 2011). However in some applications velocity dependent quadratic term is enough to describe the damping effect:

$$
\left|F_{f}\right|=K_{d} \dot{x}^{2} .
$$

Hence the dynamics of the hydraulic actuator is given by a forth order system of nonlinear differential equation defined by the relations (4), (2) and (1).

\subsection{Aerodynamic Force as Load}

Assume that the actuator drives a control surface of an aircraft, and accordingly the load in the system is represented by the aerodynamic force that acts on the control surface (see Fig. 1)

The aerodynamic force that acts on the control surface represents the load force for the actuator. Beside the actuator position, it depends on many actuator independent factors and it can hardly be measured during aircraft operation. It also depends on the sign of actuator velocity: when the control surface approaches zero position the aerodynamic force acts as a helping force; when the surface departs from zero position the force acts as a breaking force. Generally $F_{\text {aero }}$ can be considered as an unmeasurable input disturbance that depends on the states of the actuator and on external parameters as well.

The dependency of $F_{\text {aero }}$ on actuator position output and external effects in the following form can be assumed:

$F_{\text {aero }}(x)=K_{\text {aero }}(p, x, \dot{x})|x|^{\alpha(p, \dot{x})} \operatorname{sgn}(x) \operatorname{sgn}(\dot{x})+F_{0}(p) .(6)$

Here $F_{0}$ is the value of $F_{\text {aero }}$ in the zero position (when the control surface is in line with the wing), the vector $p$ incorporates mainly unmeasurable and actuator independent parameters and variables such as the aircraft altitude and speed, wind parameters, angle of attack of the airplane. $K_{\text {aero }}$ is a state dependent, time varying nonlinear gain. $\alpha$ is a parameter dependent exponent. It is considered that $\operatorname{sgn}(0)=0$.

\subsection{First Order Actuator Model for Aircraft Control Applications}

The degree of the actuator model presented above is 4. When it has to be incorporated into the model of large systems, such as aircraft models, it will substantially 
increase the degree and the complexity of the model. It is why, for controller or fault detector design, the development of a reduced order model is advisable with lover computational costs.

In order to obtain a simplified model for the electrohydraulic actuator, assume the following model simplifications:

$$
\begin{aligned}
& \ddot{x}=0, \\
& \Delta \dot{P}=0, \\
& \dot{x}_{S}=0 .
\end{aligned}
$$

Based on the equations (1), (2), (3) and (4) these simplifications lead to

$$
\begin{aligned}
& x_{S}=i, \\
& S \dot{x}=C_{v} x_{S} \sqrt{\frac{2}{\rho}\left(\Delta P_{\text {ext }}-\Delta P\right),} \\
& S \Delta P=F_{f}+F_{\text {aero }} .
\end{aligned}
$$

It is assumed that the load is represented by the aerodynamic force: $F_{L}=F_{a e r o}$.

From the equations above results

$$
\dot{x}=\sqrt{\frac{2}{\rho}} \frac{C_{v}}{S} i \sqrt{\Delta P_{e x t}-\frac{F_{f}+F_{a e r o}}{S}} .
$$

In industrial practice the model (13) is normalized with a constant positive differential pressure $\left(\Delta P_{\text {ref }}\right)$, by dividing the right hand side of the equation with $\sqrt{\Delta P_{\text {ref }}}$. The value of $\Delta P_{r e f}$ is usually taken as the pressure differential when the hydraulic valve is fully opened. The normalized model takes the form:

$$
\dot{x}=K_{c i} i \sqrt{\frac{\Delta P_{e x t}-\frac{K_{d} \dot{x}^{2}+F_{\text {aero }}}{S}}{\Delta P_{\text {ref }}}},
$$

where $K_{c i}=\sqrt{\frac{2}{\rho}} \frac{C_{v}}{S} \sqrt{\Delta P_{r e f}}$. The relation (5) was applied to describe the damping effects $\left(F_{f}\right)$ in the actuator.

The control input can be calculated in function of the prescribed position $u$ and measured position $x$ with a proportional algorithm as:

$$
i=K_{p}(u-x),
$$

where $K_{p}>0$ is the proportional gain.

The right hand side of the equation above depends trough the damping term $\left(F_{f}\right)$ on the velocity $(\dot{x})$. This dependency can be eliminated since quadratic damping term was assumed in the model. Rewrite (14) as:

$$
\frac{\dot{x}^{2} \Delta P_{r e f}}{K_{c i}^{2} i^{2}}=\Delta P_{e x t}-\frac{K_{d} \dot{x}^{2}+F_{\text {aero }}}{S} .
$$

Since $K_{d}>0$ the modified actuator model results as:

$$
\dot{x}=K_{c i} i \sqrt{\frac{\Delta P_{e x t}-\frac{F_{a e r o}}{S}}{\Delta P_{r e f}+\frac{K_{d} K_{c i}^{2} i^{2}}{S}}} .
$$

The model (17) combined with the control law (15) is widely used for designing failure detection algorithms for commercial aircrafts (Garcia et al., 2009; Lavigne et al., 2008; Goupil, 2007).

\section{ENERGY BALANCE BASED FAULT DETECTION}

In this Section we address the detection problem of control surface disconnection type fault. It can occur due to the broken linkage between the control surface and the actuator or due to the loss of the control surface. The fault can severely influence the maneuverability of the aircraft.

In industrial practice the detection of this fault can easily be solved when the control surface and the hydraulic actuator's rod are equipped with different position sensors. When the fault occurs the two sensors show different values, and accordingly the residual can be calculated as the absolute difference between the sensor outputs.

However some control surface actuation systems are equipped with only one actuator rod position sensor. In this case model based fault detection has to be applied. It can be exploited that in the case of control surface disconnection the aerodynamic force does not affect the dynamics of the actuator anymore. Based on (17) and (15) the Fault Model can be built by neglecting the load from the dynamics:

$$
\begin{aligned}
& \dot{x}_{M}=K\left(u, x_{M}\right)\left(u-x_{M}\right), \\
& K\left(u, x_{M}\right)=K_{c i} K_{p} \sqrt{\frac{\Delta P_{e x t}}{\Delta P_{r e f}+\frac{K_{d} K_{c i}^{2} K_{p}^{2}\left(u-x_{M}\right)^{2}}{S}}} .
\end{aligned}
$$

Here $x_{M}$ denotes the model output.

Based on the output of the model above, the residual signal can simply be formulated as:

$$
r=\left|x-x_{M}\right|
$$

where $x$ is the measured output of the actuator.

However, especially in the cases when during the controlled actuator motion the difference between the actual trim position and the new reference position is small, the difference between the model output and the real output can be of the order of the position sensor precision. Moreover the measurement noises can also compromise the reliability of the residual generator. Due to these reasons in this work a novel approach of for control surface disconnection detection is proposed based on the energy intake of the actuator.

Consider a Single Input Single Output mechanical system. The control input force is denoted by $F_{I N}$ and position output is denoted by $x$. Let the energy of the system be $E=E(x, \dot{x})$. With these notations the following equality (energy balance) always stands:

$$
E(t)-E\left(t_{0}\right)-\int_{t_{0}}^{t}\left(\sum F_{i}+F_{I N}\right) \dot{x} d \tau=0,
$$

where the term $\sum F_{i}$ summarizes here the external and dissipative forces acting on the mechanical system.

The energy balance of control systems has been successfully applied for control design. Control algorithms can be developed to shape the energy of the control system, see 
for example the review paper (Ortega et al., 2001). The monitoring of input energy - stored energy - dissipated energy balance of the system can also be applied for fault detection. The presence of fault disturbs the balance of the control system, for example by dissipating or injecting extra energy. By investigating the deviation of the equation (20) from zero, the fault can be detected and identified.

In an electro-hydraulic actuator the control input force is generated by the chamber pressure difference $(\Delta P)$. The pressure difference is generated by the hydraulic fluid flow $\left(Q_{f}\right)$ through the actuator. The hydraulic fluid flow depends on the externally generated pressure $\left(\Delta P_{\text {ext }}\right)$ and it is proportional with the opening of the servo valve $x_{S}$, that can be approximated with the control input $i$ of the actuator (see the relation (10)). Hence the following approximation for the force control input is reasonable: $F_{I N}=K_{I N} i=K_{I N} K_{p}(u-x)$, here $K_{I N}$ depends on $\Delta P_{\text {ext }}$.

The external and dissipative forces $\left(F_{i}\right)$ in the case of the hydraulically driven control surface are the friction force $F_{f}$ and the aerodynamic force $F_{\text {aero }}$.

Accordingly, the energy balance of the actuator during fault free case reads as:

$$
E(t)-E\left(t_{0}\right)=\int_{t_{0}}^{t}\left(F_{f}+F_{\text {aero }}+K_{I N} K_{p}(u-x)\right) \dot{x} d \tau \text {. }
$$

The energy supply of the actuator control system is:

$$
E_{S}=\int_{t_{0}}^{t} K_{I N} K_{p}(u-x) \dot{x} d \tau
$$

When a control surface disconnection occurs the aerodynamic force will no longer influence the energy balance of the actuator. Accordingly in faulty case the energy balance is:

$$
E_{F}(t)-E_{F}\left(t_{0}\right)=\int_{t_{0}}^{t}\left(F_{f}+K_{I N} K_{p}(u-x)\right) \dot{x} d \tau .
$$

Now consider the model (18), with the same position reference input $u$. The energy balance of the Fault Model reads:

$$
E_{M}(t)-E_{M}\left(t_{0}\right)=\int_{t_{0}}^{t}\left(F_{f}+K_{I N} K_{p}\left(u-x_{M}\right)\right) \dot{x}_{M} d \tau
$$

Since during faulty operation mode the model (18) describes precisely the real behavior of the actuator and the real actuator and the Fault Model have the same input $(u)$, yields $x \rightarrow x_{M}, \dot{x} \rightarrow \dot{x}_{M}$ and accordingly $E_{F} \rightarrow E_{M}$, $F_{f}(\dot{x}) \rightarrow F_{f}\left(\dot{x}_{M}\right)$. By considering that

the fault occurs in the time instant $t_{f}$ and for $t_{F}>t_{f}$ stands $x \approx x_{M}, \dot{x} \approx \dot{x}_{M}, E_{F} \approx E_{M}, F_{f}(\dot{x}) \approx F_{f}\left(\dot{x}_{M}\right)$, the input energy difference between the real actuator and the Fault Model will be constant. The supplied energy difference between the real actuator $\left(E_{S}\right)$ and the Fault Model $\left(E_{S_{M}}\right)$ can be expressed from the relations (21) and (24)

$$
\begin{aligned}
& \Delta E_{S}(t)=E_{S}(t)-E_{S_{M}}(t) \\
& =E_{F}(t)-E\left(t_{0}\right)-\int_{t_{0}}^{t_{F}}\left(F_{f}+F_{\text {aero }}\right) \dot{x} d \tau-\int_{t_{F}}^{t} F_{f} \dot{x} d \tau \\
& -\left(E_{M}(t)-E_{M}\left(t_{0}\right)-\int_{t_{0}}^{t_{F}} F_{f} \dot{x}_{M} d \tau-\int_{t_{F}} F_{f} \dot{x}_{M}\right) \\
& =E_{M}\left(t_{0}\right)-E\left(t_{0}\right)-\int_{t_{0}}^{t_{F}}\left(F_{f}+F_{\text {aero }}\right) \dot{x} d \tau-\int_{t_{0}}^{t_{F}} F_{f} \dot{x}_{M} d \tau \\
& =\text { constant, for } t>t_{F} .
\end{aligned}
$$

The input energy depends on the actuator's rod velocity. The position measurement for a real control surface actuator is highly compromised by noise hence the actuator velocity cannot be directly calculated based on the numerical differentiation of the position. Based on the Fault Model (18) the input energy in faulty case can be calculated as:

$$
\begin{aligned}
\Delta E_{S}(t) & =\int_{t-T}^{t} K(u, x) K_{I N} K_{p}(u-x)^{2} d \tau- \\
& -\int_{t-T}^{t} K\left(u, x_{M}\right) K_{I N} K_{p}\left(u-x_{M}\right)^{2} d \tau .
\end{aligned}
$$

Here $T>0$ defines the time window over which the energy difference is calculated.

Note that the formula above will not give precisely the input energy difference during faulty free operation mode since in that case the energy of the real actuator also depends on $F_{a e r o}$.

\subsection{Evaluation of the energy difference signal}

To obtain the residual signal it will be explored that $\Delta E_{S}$ given by the formula (26) is constant in faulty case but during fault free operation, assuming that the actuator is in motion, its value varies. Hence the variance of the absolute value of energy difference $\Delta E_{S}$ reads as:

$$
\begin{aligned}
r & =\operatorname{Var}\left(\left|\Delta E_{S}\right|\right) \\
& =\frac{1}{T} \int_{t-T}^{t}\left(\left|\Delta E_{S}\right|-\frac{1}{T} \int_{t-T}^{t}\left|\Delta E_{S}\right| d \tau\right)^{2} d \tau .
\end{aligned}
$$

From the relation (26) it can be seen that the variance operator is applied on a signal which contains only integral terms. Due to the low pass filter property of the integrator operator the effect of the measurement noises on the variance calculus are suppressed.

When the fault occurs $r$ given by (27) converges to zero. The decision is formulated as:

$$
d= \begin{cases}1, & \text { if } r<t h \\ 0, & \text { otherwise }\end{cases}
$$

where $t h>0$ is a small threshold value introduced to deal with model uncertainties and measurement noise during the detection process. 

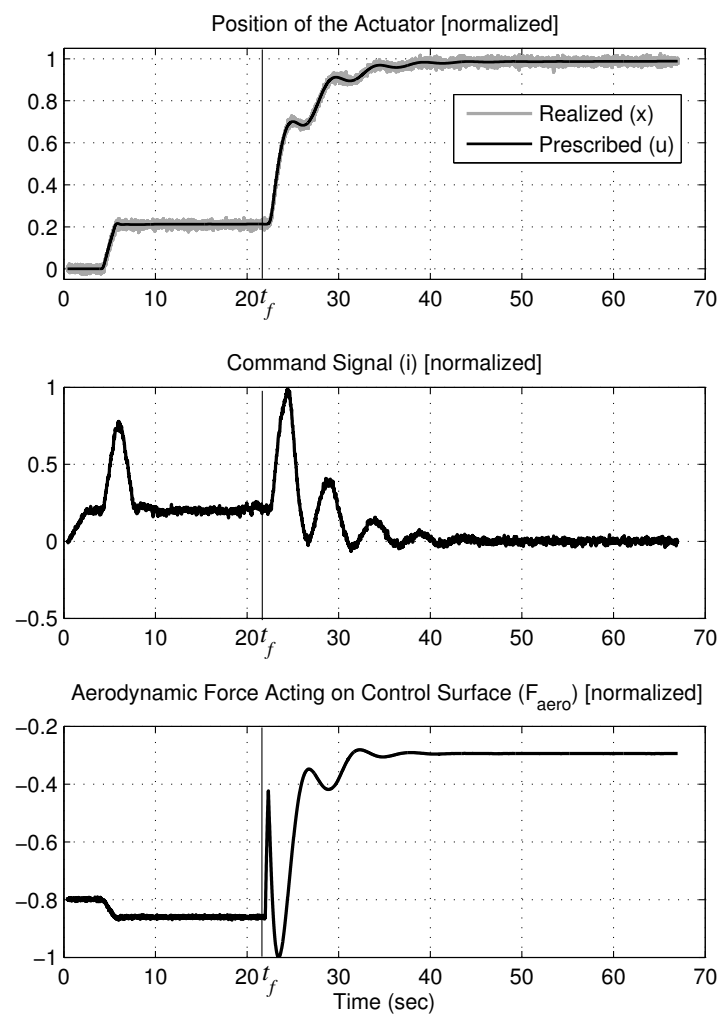

Fig. 2. The trajectory of the actuator

Remark 1: The control surface disconnection type of fault can only be detected when the actuator is in motion. In steady state (the prescribed position $u$ is constant over a long period of time) the velocity is zero and the position output of the Fault Model is equal with the measured position output. Accordingly in steady state the detector can be deactivated and the integrators can be reseted. Note that this is a physical limitation, since when an actuator is not in motion, no information from the energy consumption can be obtained from the position sensor of the actuator's rod. Hence, if the actuator is in steady state, the control surface disconnection type fault is detectable only if the behavior of the entire aircraft is observed.

Remark 2: The threshold value can be determined experimentally, by calculating the residual corresponding to the smallest prescribed position change (the resolution of the controlled motion). The residual signal is denoted as $r_{\delta}$. The threshold value should be chosen such that $0<t h<\left|r_{\delta}\right|$.

\section{SIMULATION RESULTS}

Simulations were performed in order to analyze the performances of the introduced fault detection algorithm. The method was tested on a generic civil aircraft model implemented in MATLAB/Simulink environment. The investigated control surface was a hydraulically actuated aileron.

The prescribed maximum detection time for this fault is defined as 10 seconds. It is why, for reliable fault detection,
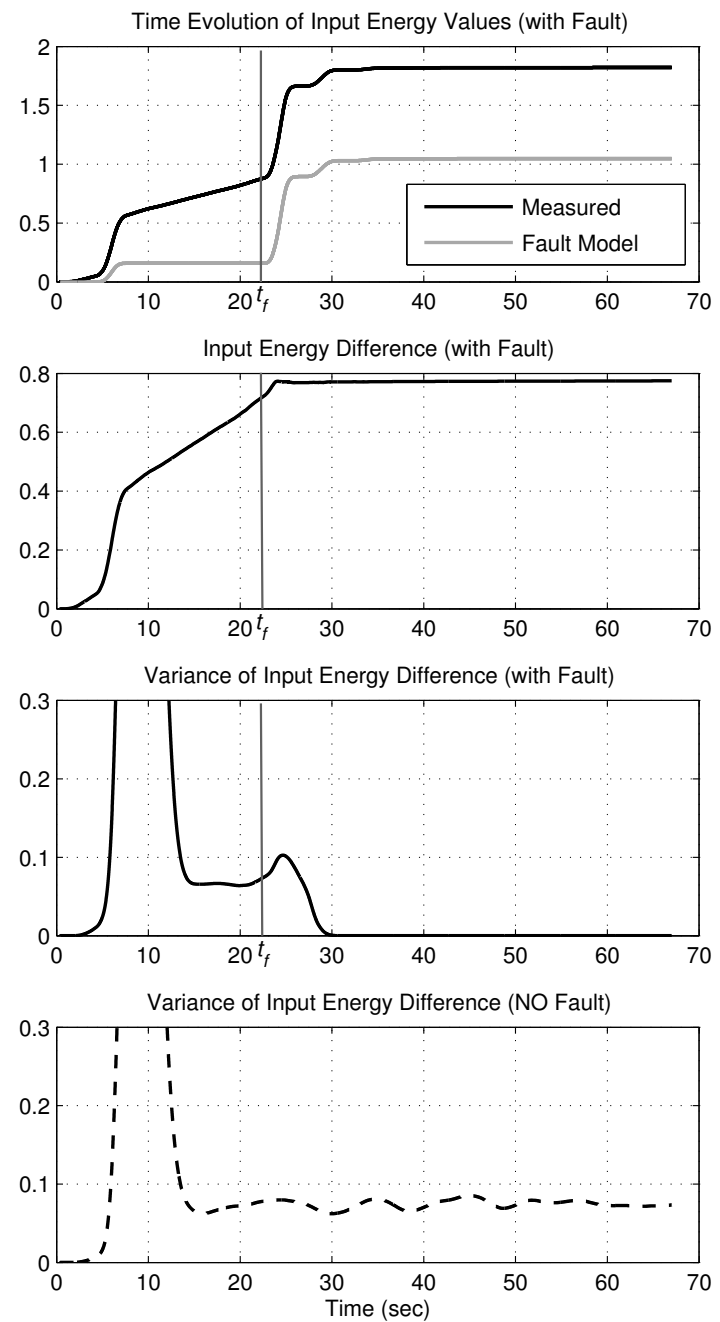

Fig. 3. Energy and residual signals

high order filters can be applied to get rid of measurement noises in the measured position signal. The time interval, over which the variance was calculated, was also set to a relatively high value (2 seconds).

Fig. 2 shows the motion of the aileron actuator's rod during a faulty flight maneuver. The prescribed actuator position output is time varying and the measured position is corrupted by high frequency measurement noise. As the figure shows, during the maneuver the difference between the prescribed and real actuator position remains under the level of the measurement noise. The fault occurs at $t_{f}=22$ seconds. Before the fault event $\left(t<t_{f}\right)$ the command signal $i$, which is proportional with the control error, does not converge precisely to zero since the controller does not contain integral term, and nonzero mean value input disturbance $\left(F_{\text {aero }}\right)$ is present in the control system. After the fault occurs $\left(t>t_{f}\right)$, the disconnected control surface goes to a position determined by the weight of the control surface and the pressure effect generated by the aerodynamic force. The aerodynamic 
force corresponding to this position is shown in the last subfigure of Fig. 1 for $t>t_{f}$.

In order to attenuate the effect of the measurement noise on the residual generation, both the actuator's position and the prescribed position were filtered using a low pass FIR (Finite Impulse Response) filter with equal filter coefficients (tap weights) and filter order 50 .

Both the filter and the fault detector were in discrete time implemented with $100 \mathrm{~ms}$ sapling period.

The difference between the input energies of the real actuator and the Fault Model (18) were calculated using the relation (26). After the fault occurrence the energy intake of the real actuator and the Fault Model is same, hence the difference converges to a constant value (see Fig. 3 ). The variance of the energy difference signal calculated using the relation (27) converges to zero, indicating that the actuator is in faulty case. Note that due to the filtering (necessary to get rid of measurement noise) the energy difference has a delayed convergence to its real value.

The experiments were repeated in normal operation (without fault occurrence) as well. As it is shown in Fig. 3 the variance of the energy difference signal will not converge to zero as long as the actuator is in motion.

The experiment was repeated for 324 different flight conditions. The fault detection rate was $100 \%$, the missed detection rate was $0 \%$ and the false detection rate was also $0 \%$. The average fault detection time was 7.8 seconds, the maximum detection time was 8.7 seconds which is under the defined fault detection time (10 second).

\section{CONCLUSIONS}

The monitoring of energy intake of aircraft actuators allows the development of new type of fault detection methods. In the case of the disconnected control surface fault the aerodynamic force will no longer influence the energy balance of the electro-hydraulic actuator. This fact was exploited for fault detector design.

The proposed detector uses the Fault Model of the actuator, which is derived from its physical model by neglecting the aerodynamic force. The energy intakes of the real actuator and the Fault Model are compared. It was shown that the energy intake can be calculated based only on position measurements, the estimation of the actuator's velocity is not necessary.

The designed fault detector is theoretically founded and has low implementation costs. It is also applicable in such cases when the difference between the Fault Model position output and the real actuator output is in order of the measurement noises and position sensor precision.

Simulation results show that the energy-balance based detector can reliably detect the control surface disconnection type faults in different flight conditions within the given detection time.

\section{ACKNOWLEDGMENTS}

The research work of L. Márton was supported by Alexander von Humboldt Stiftung/Foundation scholarship for post-doctoral researchers.

\section{REFERENCES}

Chen, W., Ding, S.X., Khan, A.Q., and Abid, M. (2010). Energy based fault detection for dissipative systems. In Proceedings of the 2010 Conference on Control and Fault Tolerant Systems, 47-52. Nice, France.

Fantuzzi, C. and Secchi, C. (2004). Energetic approach to parametric fault detection and isolation. In Proceedings of the 2004 American Control Conference, 5034 - 5039. Boston, Massachusetts.

Garcia, E.A. and Frank, P.M. (1997). Deterministic nonlinear observer-based approaches to fault diagnosis: A survey. Control Engineering Practice, 5(5), 663-670.

Garcia, E.A., Zolghadri, A., Goupil, P., Lavigne, L., and Simon, P. (2009). Nonlinear observer-based OFC detection for A380 aircraft. In Proceedings of the 7th IFAC Symposium on Fault Detection, Supervision and Safety of Technical Processes, 47-52. Barcelona, Spain.

Garimella, P. and Yao, B. (2005). Model based fault detection of an electro-hydraulic cylinder. In Proc. of 2005 American Control Conference. Portland, USA.

Goupil, P. (2007). Oscillatory failure case detection in the A380 electrical flight control system by analytical redundancy. In Seventeenth IFAC Symposium on Automatic Control in Aerospace. ICT, Universitas Studii Tolosana, France.

Khan, H., Abou, S.C., and Sepehri, N. (2005). Nonlinear observer-based fault detection technique for electrohydraulic servo-positioning systems. Mechatronics, 15(9), 1037-1059.

Lavigne, L., Zolghadri, A., Goupil, P., and Simon, P. (2008). Oscillatory failure case detection for new generation Airbus aircraft: a model-based challenge. In 47 th IEEE Conference on Decision and Control, 1249-1254. Cancun, Mexico.

Leuschen, M., Walker, I., and Cavallaro, J. (2003). Nonlinear Fault Detection for Hydraulic Systems, 169-191. Fault Dyagnosis and Fault Tolerance for Mechatronics Systems: Recent Advances, Springer.

Márton, L., Fodor, S., and Sepehri, N. (2011). A practical method for friction identification in hydraulic actuators. Mechatronics, 21(1), 350-356.

Merritt, H. (1967). Hydraulic control systems. John Wiley \& Sons, New York.

Ortega, R., van der Schaft, A., Mareels, I., and Maschke, B. (2001). Putting energy back in control. IEEE Control Systems Magazine, 21(2), 18-32.

Tan, H.Z. and Sepehri, N. (2002). Parametric fault diagnosis for electrohydraulic cylinder drive units. IEEE Transactions on Industrial Electronics, 49(1), 96-106.

Theilliol, D., Noura, H., Sauter, D., and Hamelin, F. (2006). Sensor fault diagnosis based on energy balance evaluation: Application to a metal processing. ISA Transactions, 45(4), 603-610.

Varga, A. (2011). On parametric solution of fault detection problems. In Proc. of 18th IFAC World Congress. Milan, Italy.

Wang, X. and Syrmos, V.L. (2008). Fault detection, identification and estimation in the electro-hydraulic actuator system using EKF-based multiple-model estimation. In Proc. of 16th Mediterranean Conference on Control and Automation. Ajaccio, France. 